\title{
Rotational inertia and multimodal heaviness perception
}

\author{
Matthew Streit, Kevin Shockley, and Michael A. Riley \\ University of Cincinnati, Cincinnati, Ohio
}

\begin{abstract}
Perceived heaviness of wielded objects has been shown to be a function of the objects' rotational inertia-the objects' resistance to rotational acceleration. Studies have also demonstrated that if virtual objects rotate faster than the actual wielded object (i.e., a rotational gain is applied to virtual object motion), the wielded object is perceived as systematically lighter. The present research determined whether combining those inertial and visual manipulations would influence heaviness perception in a manner consistent with an inertial model of multimodal heaviness perception. Rotational inertia and optical rotational gain of wielded objects were manipulated to specify inertia multimodally. Both visual and haptic manipulations significantly influenced perceived heaviness. The results suggest that rotational inertia is detected multimodally and that multimodal heaviness perception conforms to an inertial model.
\end{abstract}

Since Weber's $(1834 / 1978)$ psychophysical studies on heaviness perception, it has been known that the perceived heaviness of a hefted object does not correspond simply to the object's mass. A classic illustration is the size-weight (S-W) illusion (see, e.g., Charpentier, 1891; Cross \& Rotkin, 1975; Stevens \& Rubin, 1970). When confronted with two objects of the same mass but different size, people typically report that the larger object is lighter than the smaller object. S-W illusion studies have demonstrated that haptic and visual (e.g., Amazeen, 1997; Ellis \& Lederman, 1993) information influence heaviness perception. The nature of visual and dynamic touch contributions and their relative importance, however, remains contentious.

Amazeen and Turvey (1996) theorized that the information about heaviness obtained via dynamic touch (touch involving muscular effort as an object is actively wielded) is the rotational inertia of a wielded object - how much an object resists rotational acceleration (see also Kreifeldt \& Chuang, 1979). In its most general form, the rotational inertia $I$ of an object about a single axis can be quantified as

$$
I \approx \operatorname{mass} \times(C M-O)^{2},
$$

where $C M$ is the location of the object's center of mass and $O$ is the location of the rotation point. In Amazeen and Turvey's study, they considered the inertial properties of objects used in a comprehensive $\mathrm{S}-\mathrm{W}$ illusion investigation by Stevens and Rubin (1970). As the objects used by Stevens and Rubin increased in size, they showed a particular patterning of rotational inertia. Amazeen and Turvey used specialized objects that allowed precise control over the rotational inertia about different rotational axes. They found the following relation between perceived heaviness and rotational inertia for wielding nonvisible objects:

$$
\text { Heaviness } \mathrm{perceived}=0.31 \cdot I_{1}^{36} \cdot I_{3}^{-.12},
$$

where $I_{1}$ is effectively the rotational inertia for radialulnar flexion-extension and $I_{3}$ is effectively the rotational inertia for pronation/supination. Equation 2 states that as $I_{1}$ increases and as $I_{3}$ decreases, the object is perceived as heavier (for other inertia models, see Amazeen, 1997; Shockley, Carello, \& Turvey, 2004; Shockley, Grocki, Carello, \& Turvey, 2001; Turvey, Shockley, \& Carello, 1999). What Amazeen and Turvey discovered, more generally, was that previously demonstrated influences of object size on perceived heaviness were not actually due to changes in size, per se, but instead to changes in rotational inertia that accompanied changes in size.

Importantly, however, heaviness can also be perceived via vision alone. Runeson and Frykholm (1981) demonstrated that perceivers could accurately judge the heaviness of a box by watching a point-light display of another person lifting the box. Similarly, Bingham (1987) showed that the heaviness of a lifted object could be perceived by observing point-light displays of the lifter's arm curl. The implication of these studies is that visual kinematic information alone is sufficient to influence heaviness perception, even when the perceiver is not touching the object. This general availability of information about dynamics (i.e., forces and masses) from kinematics (motion) was termed by Runeson the kinematic specification of $d y$ namics (KSD) principle (Runeson, 1977/1983; Runeson \& Frykholm, 1981, 1983; Runeson, Juslin, \& Olsson, 2000). 
Streit, Shockley, Morris, and Riley (2007; see also Streit $\&$ Shockley, 2005) used the KSD principle to link inertial models of heaviness perception to visual perception of heaviness. Their approach rests on the fact that when an object rotates in response to an applied torque $N$ the dynamics (i.e., $N$ and the object's inertia, $I$ ) and kinematics (i.e., the object's rotational acceleration, $\dot{\omega}$ ) are causally linked. This relation is approximated (Amazeen \& Turvey, 1996; Fitzpatrick, Carello, \& Turvey, 1994) ${ }^{1}$ as

$$
N=I \dot{\omega},
$$

which can be rewritten as

$$
I=\frac{N}{\dot{\omega}}
$$

Following from the KSD principle, Streit et al. (2007) reasoned that a change in the apparent responsiveness (i.e., $\dot{\omega})$ of an object to a given applied muscular torque would specify a change in the rotational inertia $I$. Thus, if multimodal heaviness perception is a function of the specified rotational inertia of a wielded object, then changing the apparent responsiveness of a given object to applied forces should influence heaviness perception accordingly. To test that hypothesis, they had participants wield objects while watching virtual representations of the objects that moved in real-time with the physical objects. A scaling factor-rotational gain - was applied to the virtual object's sagittal-plane motion such that the virtual object rotated more or less quickly (changing $\dot{\omega}$ ) relative to its actual wielded counterpart. Streit et al. found that perceived heaviness was inversely proportional to rotational gain (see also Streit \& Shockley, 2005) and was not influenced by nonspecifying cues such as wrist rotation amplitude or virtual object rotation amplitude.

Neither Streit et al. (2007) nor Streit and Shockley (2005) manipulated the actual rotational inertia of the objects. Such a manipulation is important for two reasons. First, it is necessary to simultaneously manipulate optical rotational gain and rotational inertia in order to rule out the possibility that perceptual reports were influenced by rotational gain simply because that was the only aspect of the objects that changed.

Second, manipulating only rotational gain does not permit an examination of how visual and dynamic touch influences combine, an issue that remains contentious. Stevens and Rubin (1970) and Cross and Rotkin (1975) suggested that perceived heaviness is dependent on object volume (i.e., percept-percept coupling), indicating perceptual dependence of touch perception on visual perception (Epstein, 1982). However, Amazeen (1999) and Riley and Turvey (2001) found that visual and touch influences were additive, suggesting perceptual independence (see also Grandy \& Westwood, 2006). Hay, Pick, and Ikeda (1965) further suggested that vision dominates touch when the two modalities are discrepant, though later studies revealed that the touch information is never lost (e.g., Pick, Warren, \& Hay, 1969; Warren \& Pick, 1970).

The present research was designed to rule out the first possibility and to assess the relative contributions of visual and dynamic touch inertial information on perceived
Table 1

Rotational Inertia of the Experimental Objects and the Corresponding Multimodally Specified Rotational Inertia Derived From Equation A5 for the Four Rotational Gain Levels

\begin{tabular}{lccc}
\hline Object & $\begin{array}{c}\text { Actual } \\
\text { Rotational Inertia } \\
\left(I_{1}\right)\left(\mathrm{kg} \cdot \mathrm{m}^{2}\right)\end{array}$ & $\begin{array}{c}\text { Rotational } \\
\text { Gain }(\times \text { actual } \\
\text { rotation })\end{array}$ & $\begin{array}{c}\text { Multimodally Specified } \\
\text { Rotational Inertia } \\
\left(I_{\text {specified }}\right)\left(\mathrm{kg} \cdot \mathrm{m}^{2}\right)\end{array}$ \\
\hline$I_{\text {low }}$ & 0.020217 & 0.7 & 0.028881 \\
& & 0.9 & 0.022463 \\
$I_{\text {medium }}$ & 0.027825 & 1.1 & 0.018379 \\
& & 1.3 & 0.015552 \\
& & 0.7 & 0.039750 \\
$I_{\text {high }}$ & 0.039622 & 1.1 & 0.030917 \\
& & 1.3 & 0.025295 \\
& & 0.7 & 0.021404 \\
& & 0.9 & 0.056603 \\
& & 1.1 & 0.044024 \\
& & 1.3 & 0.036020 \\
& & &
\end{tabular}

heaviness. In doing so, the research offers to provide insight into the informational basis of multimodal heaviness perception. We propose that multimodal heaviness perception is specific to multimodally specified inertia - the ratio of applied torque to optical rotational acceleration. Multimodally specified inertia ( $\left.I_{\text {specified }}\right)$ can be empirically derived using Equation 4 (see the Appendix for the derivation and Table 1 for multimodally specified inertia magnitudes as a function of rotational gain). As illustrated in Figure 1, multimodally specified inertia can be used to predict perceived heaviness by substituting $I_{\text {specified }}$ for $I_{1}$ in Equation 2. Thus, if inertia is detected multimodally, the results should pattern according to Figure 1. Such a finding would provide strong evidence that multimodal heaviness perception conforms to inertia models of heaviness perception.

\section{METHOD}

\section{Participants}

Seventeen University of Cincinnati undergraduates (12 women, 5 men; $18-45$ years old, $M=23.4$ ) participated to fulfill a course requirement. All participants were right-handed by self-report.

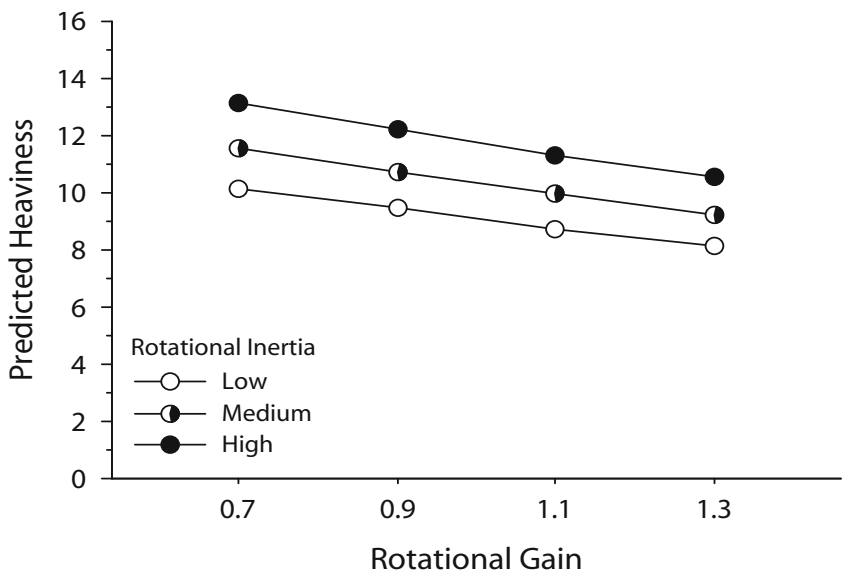

Figure 1. Predicted perceived heaviness from Equation 2 using $I_{\text {specified }}$ from Table 1 in place of $I_{1}$. 


\section{Apparatus}

Participants sat in a wooden chair facing a wooden frame. The frame was covered by an occluding curtain containing two slits into which participants placed their hands. A padded support was attached to the frame at arm level, allowing participants to rest their forearms.

The wielded objects consisted of a cylindrical wooden handle $(20.5 \mathrm{~cm} \times 3 \mathrm{~cm})$ with an inserted wooden $\operatorname{rod}(69 \mathrm{~cm} \times 1.25 \mathrm{~cm})$ and a 100 -g fiberglass mass attached to the rod. The mass was located on the rod 16,28 , or $40 \mathrm{~cm}$ from the distal end of the handle, to yield $I_{1}$ values of $202,170 \mathrm{~g} \cdot \mathrm{cm}^{2}\left(I_{\text {low }}\right), 278,250 \mathrm{~g} \cdot \mathrm{cm}^{2}\left(I_{\text {medium }}\right)$, or $396,220 \mathrm{~g} \cdot \mathrm{cm}^{2}\left(I_{\text {high }}\right) . I_{3}$ remained constant at $3,963 \mathrm{~g} \cdot \mathrm{cm}^{2}$ for all objects.

A projection screen was located $290 \mathrm{~cm}$ away and $45^{\circ}$ to the participant's left. Virtual renderings of the wielded objects were projected onto the screen using an Epson PowerLite S3 projector (1600 ANSI lumens). A 6-D motion-tracking sensor (FasTrak II; Polhemus, Inc., Colchester, VT; sampling rate of $24 \mathrm{~Hz}$ ) was attached to the base of each object's handle. Motion data were fed to an IBM T40 Thinkpad (Pentium M, $1.6 \mathrm{gHz}, 1 \mathrm{gB}$ RAM) via the serial port. The computer generated the virtual objects (see Figure 2). Data collection and virtual object rendering were managed by customized MATLAB 7.0 (The MathWorks, Inc., Natick, MA) routines.

One object served as the standard $\left(I_{\text {medium }}\right)$. During instruction, the other (test) object corresponded to $I_{\text {medium }}$. During experimental trials, test objects corresponded either to $I_{\text {low }}, I_{\text {medium }}$, or $I_{\text {high }}$. The projection screen displayed virtual depictions of the objects. Two red spheres were also depicted, serving as targets that constrained wielding amplitude (Figure 2A). One of the targets was always positioned directly above the base of the virtual object. The second target position varied randomly across trials between $30^{\circ}$ and $50^{\circ}$. The virtual depiction of the standard was displayed in green, and the test object in blue (different colors were used so participants would not confuse the two). The virtual objects moved in real time with the actual objects. A scaling factor (rotational gain $=0.7,0.9,1.1$, or $1.3 \times$ actual object rotation; see Figure $2 \mathrm{~B}$ ) was applied to the virtual test object's rotational motion. The scaling factor changed the amplitude and, therefore, the velocity and acceleration of the virtual object's excursions relative to the actual object (see the Appendix).

\section{Procedure}

Participants sat in the wooden chair and placed each hand through a slit in the curtain. The standard object was placed in one hand and a test object was placed in the other. The hand into which the standard and test objects were placed was randomized across participants. A trial consisted of a participant first wielding the standard back and forth between the targets until an impression of heaviness was obtained. The participant then wielded the test object in the same manner. Participants were allowed to alternate between wielding the standard and test object as many times as desired, but to avoid direct comparison of rotation angles they were instructed to only wield one object at a time. Participants then reported the heaviness of the test object relative to the standard of 100 arbitrary units. After making a report, participants oriented both objects vertically (aligned with the fixed target), and the experimenter advanced the virtual display to the next rotational gain setting. The experimenter then firmly grasped the test object handle (to prevent the participant from wielding it), removed the inserted wooden rod from the handle of the test object, placed it aside, and inserted the appropriate rod for the next trial into the handle. The experimenter then released the test object handle.

The experiment consisted of four blocks (48 total trials) and lasted approximately $45 \mathrm{~min}$. One of each of the 12 possible rotational gain-rotational inertia combinations was randomly ordered within each block. At the end of the experiment, participants reported how many different objects they believed they wielded. Participants were also asked to describe any strategies they used so the experimenter could determine if they had realized the nature of the gain manipu-
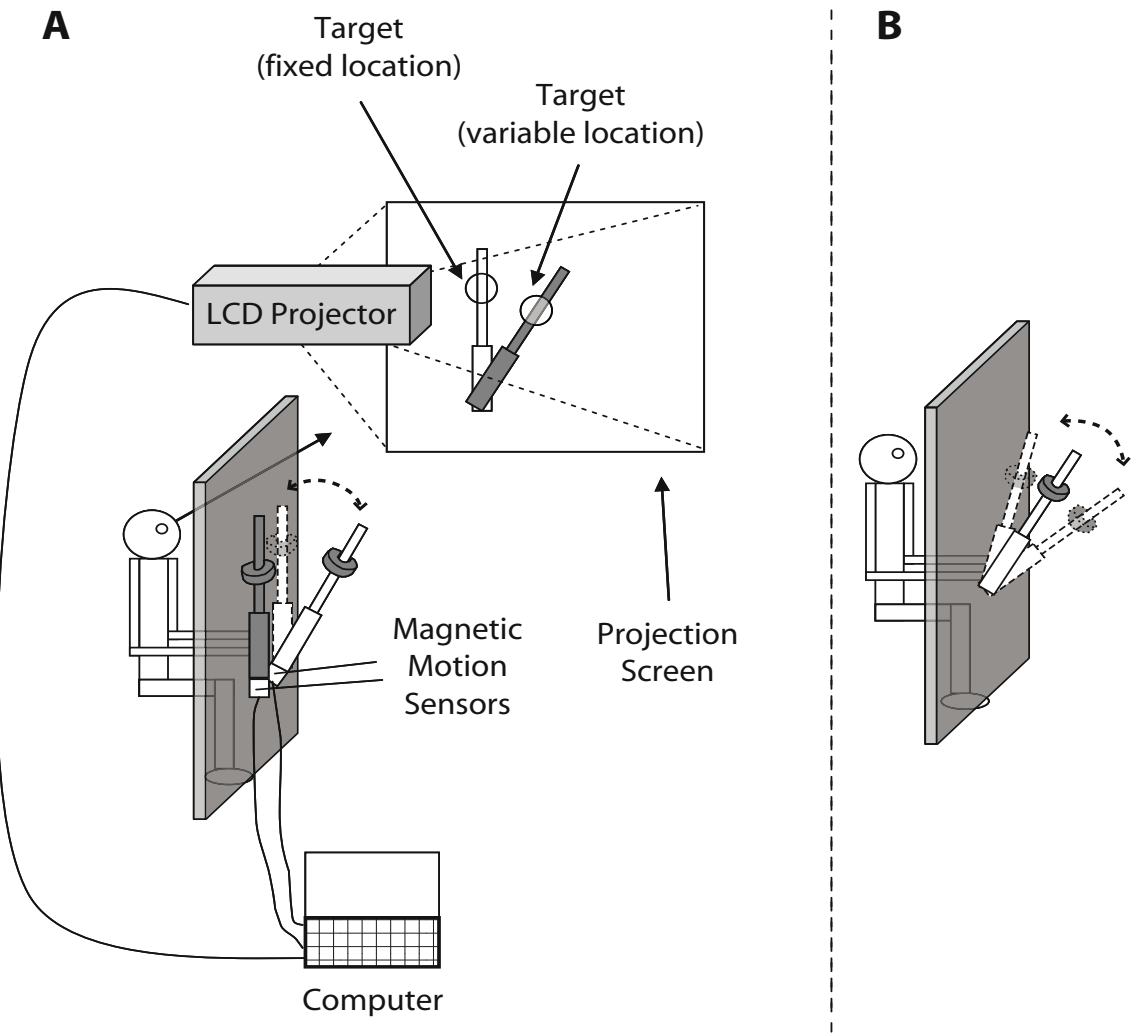

Low Rotational Gain

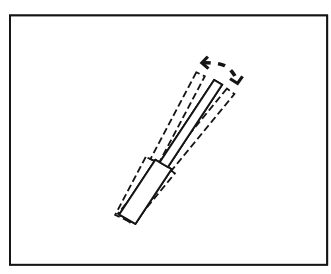

High Rotational Gain

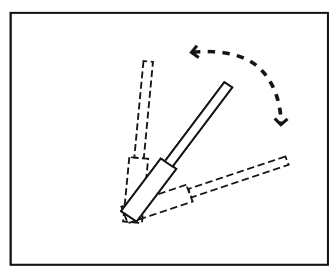

Figure 2. Illustration of (A) method and (B) rotational gain manipulation. 
lation. Procedures were approved by the University of Cincinnati Institutional Review Board.

\section{RESULTS}

Participants reported a mean of 8.1 different objects $(S D=5.1)$ (1 participant was unable to provide an estimate). None of the participants deduced the rotational gain manipulation.

Mean heaviness reports for each condition for each subject were submitted to a 3 (rotational inertia) $\times 4$ (rotational gain) repeated measures ANOVA with a HuynhFeldt adjustment. As illustrated in Figure 3A, there were significant main effects of rotational inertia $[F(1.11$, $\left.17.81)=34.332, p<.01, \eta_{\mathrm{p}}^{2}=.68\right]$ and rotational gain $\left[F(1.22,19.47)=14.29, p<.01, \eta_{\mathrm{p}}^{2}=.47\right]$. There was no significant interaction $\left(F<1, \eta_{\mathrm{p}}^{2}=.04\right)$.

The 12 mean heaviness values were submitted to a multiple linear regression with rotational inertia and rotational gain as predictors. The regression accounted for $98 \%$ of the variance and yielded the following equation: Heaviness perceived $=92.53+2.614 \cdot($ rotational inertia $)-$ $48.63 \cdot$ (rotational gain). ${ }^{2}$ Rotational inertia accounted for $78 \%$ of the total variance $\left(\beta_{\text {inertia }}=.88, p<.01\right)$ and rotational gain accounted for $21 \%$ of the total variance $\left(\beta_{\text {gain }}=-.459, p<.01\right)$.

As shown in Figure 3B, mean perceived heaviness values were regressed onto the predicted heaviness values (derived from Equation 2 using multimodally specified inertia values in Table 1 in place of $I_{1}$ ) to determine how well the present results conformed to predictions from the Amazeen and Turvey (1996) inertia model. The regression equation was Heaviness perceived $=16.55$. (predicted heaviness) -52.69 . Predicted heaviness accounted for $95 \%$ of the variance in perceived heaviness. Note that Amazeen and Turvey used a standard of 10 as compared to the present standard of 100 , so the slope and y-intercept of the regression equation reflect this order-of-magnitude difference.

\section{DISCUSSION}

The present study revealed that heaviness perception is influenced by both optical rotational gain and rotational inertia. As predicted, perceived heaviness was positively related to rotational inertia and inversely related to rotational gain. The standardized regression coefficient for rotational inertia (.88) was almost double that for rotational gain (-.459), suggesting at first glance that the influence of rotational gain was attenuated by concurrent manipulations of rotational inertia. However, it is important to note that the inertial manipulations were also larger in magnitude than the gain manipulations. That is, the average $I_{\text {specified }}$ change $\left(0.0193 \mathrm{~kg} \cdot \mathrm{m}^{2}\right)$ was only $66 \%$ of the average $I_{1}$ change $\left(0.0292 \mathrm{~kg} \cdot \mathrm{m}^{2}\right)$. Thus, the difference in standardized regression coefficients may reflect the magnitude of the respective manipulations rather than the relative importance of each factor for perception. It should be further noted that the visual depictions of the wielded objects were highly artificial — a simple computer-generated display in a third-person perspective - as opposed to the normal first-person view of wielding a real rod in one's own hand. Thus, any visual influence may have been attenuated by the artificial nature of the display. However, the suggestion that touch information has a stronger influence than visual information is supported by the fact that Streit et al. (2007) found that rotational gain accounted for $98 \%$ of the variance in perceived heaviness, while in the present study rotational gain accounted for only $21 \%$ of the total variance (though both studies have approximately the same coefficient for rotational gain). A stronger test of the relative contributions of the two modalities requires manipulating $I_{1}$ and $I_{\text {specified }}$ by identical magnitudes.

Although dynamic touch and visual influences were both significant and did not interact, this does not necessarily imply that those influences correspond to two, distinct percepts (rotational inertia detected via deformations of muscles/tendons and visually detected kinematics) that influence heaviness perception independently. Stoffregen and Bardy (2001) suggested that perception is specific to information that exists in irreducible, higher-order patterns across different forms of stimulus energy. They further argued that the various perceptual modalities function together as a single unit in detecting information patterns that extend across different energetic media. As outlined in the
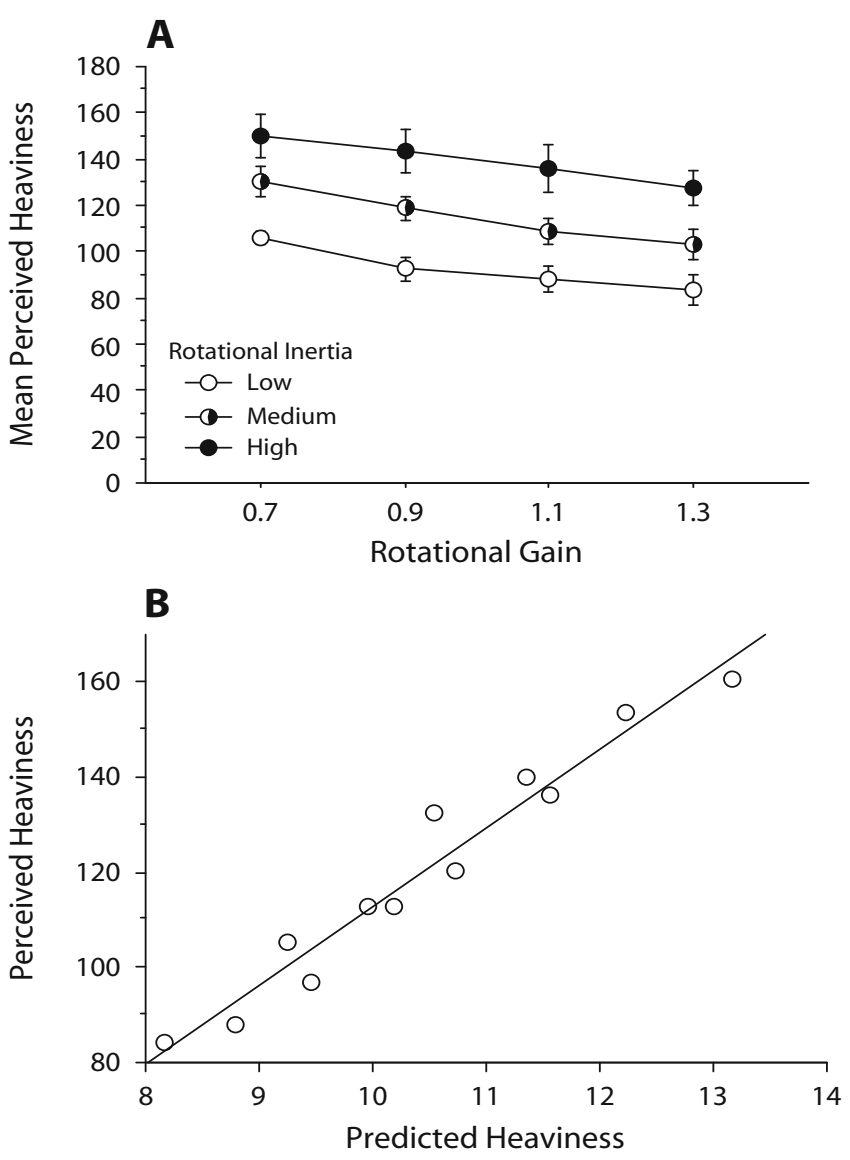

Figure 3. (A) Mean perceived heaviness as a function of rotational inertia and rotational gain. Error bars reflect one standard error. (B) Perceived heaviness regressed onto predicted heaviness. 
Appendix, $I_{\text {specified }}$ is defined as the relation between the actual inertia of an object and the rotational gain factor, i.e., as a relational pattern that extends across mechanical and optical media. Given the near-perfect fit between our results and those predicted by inertial models (using $I_{\text {specified }}$ in place of $I_{1}$ ), multimodal heaviness perception appears to be specific to the kinds of higher-order, relational, multimodal quantities suggested by Stoffregen and Bardy.

Our results furthermore suggest that the detection of rotational inertia is based on the relation between applied torque and the object's responsiveness. Although studies have demonstrated that dynamic touch perception is invariant across changes in wielding torque (see Amazeen \& Turvey, 1996, Experiment 2, for heaviness perception, and Pagano \& Cabe, 2003, for length perception), Greer (1989) showed an influence of an externally imposed resistance to motion on perceived heaviness of a translated object. Objects that have an externally imposed translational resistance (and, therefore, require greater force to be moved) were perceived as heavier than translated objects that had no externally imposed resistance. Thus, consistent with our position, although the object's mass (i.e., translational inertia) did not actually change in Greer's study, objects felt differentially heavy by virtue of their differential responsiveness to applied force.

The actual responsiveness of an object to applied torque could be manipulated in a manner similar to the present visual manipulation of virtual object responsiveness. For example, a robotic manipulandum could be programmed to generate rotational forces that modulate how an object would ordinarily respond to wielding torques (see Shadmehr, Mussa-Ivaldi, \& Bizzi, 1993). If rotational inertia is detected by virtue of the relation between applied torque and object responsiveness, then for heaviness perception by dynamic touch the same mapping of perceived heaviness to $I_{\text {specified }}$ should be obtained for comparable gains achieved via such a manipulation.

\section{AUTHOR NOTE}

This research was supported by National Science Foundation Grant BCS-0716319 awarded to K.S. and M.R., and by an award from the William Seeman Fund to M.S., for whom the research formed the basis for the master's thesis. We thank Anthony Morris for his technical contributions and Eric Amazeen, Christopher Pagano, and Sverker Runeson for their very helpful comments and suggestions. Correspondence relating to this article may be sent to M. Streit, Department of Psychology, ML 0376, 429 Dyer Hall, University of Cincinnati, Cincinnati, OH 45221-0376 (e-mail: streitms@email.uc.edu).

\section{REFERENCES}

Amazeen, E. (1997). The effects of volume on perceived heaviness by dynamic touch: With and without vision. Ecological Psychology, 9, 245-263.

Amazeen, E. (1999). Perceptual independence of size and weight by dynamic touch. Journal of Experimental Psychology: Human Perception \& Performance, 25, 102-119.

Amazeen, E., \& Turvey, M. T. (1996). Weight perception and the haptic size-weight illusion are functions of the inertia tensor. Journal of Experimental Psychology: Human Perception \& Performance, 22, 213-232.

Bingham, G. (1987). Kinematic form and scaling: Further investigations on the visual perception of lifted weight. Journal of Experimental Psychology: Human Perception \& Performance, 13, 155-177.
Charpentier, A. (1891). Analyse experimentale de quelques elements de la sensation de poids [Experimental study of some aspects of weight perception]. Archives de Physiologie Normale et Pathologique, 3, 122-135.

Cross, D. V., \& RotKin, L. (1975). The relation between size and apparent heaviness. Perception \& Psychophysics, 18, 79-87.

Ellis, R. R., \& Lederman, S. (1993). The role of haptic versus visual volume cues in the size-weight illusion. Perception \& Psychophysics, 53, 315-324.

EPstein, W. (1982). Percept-percept coupling. Perception, 11, 75-83.

FitzPatrick, P., Carello, C., \& Turvey, M. T. (1994). Eigenvalues of the inertia tensor and exteroception by the "muscular sense." Neuroscience, 60, 551-568.

Grandy, M. S., \& Westwood, D. A. (2006). Opposite perceptual and sensorimotor responses to a size-weight illusion. Journal of Neurophysiology, 95, 3887-3892.

GrEeR, K. (1989). Gravitational and inertial effects of mass on the perceived heaviness of objects. Bulletin of the Psychonomic Society, 27, $18-20$

HAY, J. C., Pick, H. L., JR., \& IKedA, K. (1965). Visual capture produced by prism spectacles. Psychonomic Science, 2, 215-216.

Kreifeldt, J. G., \& Chuang, M. (1979). Moment of inertia: Psychophysical study of an overlooked sensation. Science, 206, 588590.

Pagano, C. C., \& Cabe, P. A. (2003). Constancy in dynamic touch: Length perceived by dynamic touch is invariant over changes in media. Ecological Psychology, 15, 1-17.

Pick, H. L., Warren, D. H., \& Hay, J. C. (1969). Sensory conflict in judgments of spatial direction. Perception \& Psychophysics, 6, 203-205.

Riley, M. A., \& Turvey, M. T. (2001). Inertial constraints on limb proprioception are independent of visual calibration. Journal of Experimental Psychology: Human Perception \& Performance, 27, 438-455.

RUNESON, S. (1983). On the visual perception of dynamic events (Acta Universitatis Upsaliensis: Studia Psychologica Upsaliensia, Serial 9). Uppsala: University of Uppsala. (Original work published 1977)

Runeson, S., \& Frykholm, G. (1981). Visual perception of lifted weight. Journal of Experimental Psychology: Human Perception \& Performance, 7, 733-740.

Runeson, S., \& FryKholm, G. (1983). Kinematic specification of dynamics as an informational basis for person-and-action perception: Expectation, gender recognition, and deceptive intention. Journal of Experimental Psychology: General, 112, 585-615.

Runeson, S., Juslin, P., \& Olsson, H. (2000). Visual perception of dynamic properties: Cue heuristics versus direct perceptual competence. Psychological Review, 107, 525-555.

Shadmehr, R., Mussa-Ivaldi, F. A., \& Bizzi, E. (1993). Postural force fields of the human arm and their role in generating multijoint movements. Journal of Neuroscience, 13, 45-62.

Shockley, K., Carello, C., \& Turvey, M. T. (2004). Metamers in the haptic perception of heaviness and moveableness. Perception \& Psychophysics, 66, 731-742.

Shockley, K., Grocki, M., Carello, C., \& Turvey, M. T. (2001). Somatosensory attunement to the rigid body laws. Experimental Brain Research, 136, 133-137.

Stevens, J. C., \& Rubin, L. L. (1970). Psychophysical scales of apparent heaviness and the size-weight illusion. Perception \& Psychophysics, 8, 225-230.

Stoffregen, T. A., \& BARdy, B. G. (2001). On specification and the senses. Behavioral \& Brain Sciences, 24, 195-261.

Streit, M., \& Shockley, K. (2005). Optical gain and the perception of heaviness. In H. Heft \& K. L. Marsh (Eds.), Studies in perception and action VIII (pp. 185-187). Mahwah, NJ: Erlbaum.

Streit, M., Shockley, K., Morris, A., \& Riley, M. A. (2007). Rotational kinematics influence multimodal perception of heaviness. Psychonomic Bulletin \& Review, 14, 363-367.

Turvey, M. T., Shockley, K., \& Carello, C. (1999). Affordance, proper function and the physical basis of perceived heaviness. Cognition, 73, B17-B26.

WARREN, D. H., \& PICK, L. (1970). Intermodality relations in localization in blind and sighted people. Perception \& Psychophysics, 8, 430-432.

Weber, E. H. (1978). The sense of touch (H.E. Ross, Ed. \& Trans.). London: Academic Press. (Original work published 1834) 


\section{NOTES}

1. A more complete characterization of the relation between torque $(N)$, rotational inertia $(I)$, and rotational acceleration $(\dot{\omega})$ is given by

$$
N_{1}=I_{1} \dot{\omega}_{1}-\omega_{2} \omega_{3}\left(I_{2}-I_{3}\right),
$$

where $\omega$ corresponds to angular velocity, and subscripts 1,2 , and 3 correspond to the three spatially orthogonal symmetry axes (eigenvec- tors) dictated by the mass distribution about a given point of rotation. Because radial-ulnar flexion-extension approximates rotation about axis $1, \omega_{2}$ and $\omega_{3}$ approach zero. Thus, this relation effectively reduces to Equation 3.

2. The units for rotational inertia are typically $\mathrm{g} \cdot \mathrm{cm}^{2} \mathrm{or} \mathrm{kg} \cdot \mathrm{m}^{2}$. Because the first would result in a very small regression coefficient, and the second would result in a very large regression coefficient, rotational inertia units of $\mathrm{g} \cdot \mathrm{m}^{2}\left(\mathrm{~g} \cdot \mathrm{cm}^{2} / 10,000\right)$ were used in the regression.

\section{Calculation of Multimodally Specified Inertia}

Angular displacement $(\theta)$ of a an object about a given rotational axis can be quantified as

$$
\theta=\theta_{0}+\omega_{0}+\frac{N}{2 I} t^{2}
$$

where $\theta_{0}$ is the initial angular displacement, $\omega_{0}$ is the initial angular velocity, $N$ is applied torque, $I$ is rotational inertia, and $t$ is time. The corresponding apparent angular displacement $\left(\theta_{\text {apparent }}\right)$ of the virtual object - the angular displacement resulting from an applied rotational gain — can therefore be quantified as

$$
\theta_{\text {apparent }}=\theta_{0}+\omega_{0}+\left(\frac{N}{2 I} t^{2}\right) \cdot \text { RotationalGain, }
$$

where $\theta_{0}$ and $\omega_{0}$ are zero. Apparent angular velocity $\left(\omega_{\text {apparent }}\right)$ corresponds to the first time derivative of $\theta_{\text {apparent }}$,

$$
\omega_{\text {apparent }}=\frac{d \theta_{\text {apparent }}}{d t}=\frac{N \cdot \text { RotationalGain }}{I} t,
$$

and apparent angular acceleration $\left(\dot{\omega}_{\text {apparent }}\right)$ is the first time derivative of $\omega_{\text {apparent }}$,

$$
\dot{\omega}_{\text {apparent }}=\frac{d \omega_{\text {apparent }}}{d t}=\frac{N \cdot \text { RotationalGain }}{I} .
$$

Given $\dot{\omega}_{\text {apparent }}$, multimodally specified inertia ( $\left.\mathrm{I}_{\text {specified }}\right)$ may then be derived via Equation 4:

$$
I_{\text {specified }}=\frac{N}{\dot{\omega}_{\text {apparent }}}=\frac{I}{\text { RotationalGain }} \text {. }
$$

(Manuscript received August 22, 2006;

revision accepted for publication December 4, 2006.) 